Mots. Les langages du politique

Suisse, laboratoire politique européen ?

\title{
La constitution du public dans la démocratie semi- directe.Le cas de l'initiative populaire « pour une procréation respectant la dignité humaine »
}

Alain Bovet

\section{CpenEdition}

Journals

Édition électronique

URL : https://journals.openedition.org/mots/650

DOI : $10.4000 /$ mots.650

ISSN : 1960-6001

Éditeur

ENS Éditions

Édition imprimée

Date de publication : 1 juillet 2006

Pagination : 35-48

ISBN : 2-84788-098-4

ISSN : 0243-6450

Référence électronique

Alain Bovet, « La constitution du public dans la démocratie semi-directe.Le cas de l'initiative populaire " pour une procréation respectant la dignité humaine » », Mots. Les langages du politique [En ligne], 81 2006, mis en ligne le 01 juillet 2008, consulté le 23 avril 2022. URL : http://journals.openedition.org/ mots/650 ; DOl : https://doi.org/10.4000/mots.650 


\section{La constitution du public dans la démocratie semi- directe. Le cas de l'initiative populaire « pour une procréation respectant la dignité humaine "}

Selon les conventions de la démocratie suisse, le citoyen est invité plusieurs fois par an à se prononcer sur des objets qui engagent profondément le devenir du pays. Il peut ainsi contribuer à modifier la Constitution ou à invalider des décisions du Parlement. L'expression démocratie semi-directe est généralement employée pour désigner cette forme développée de participation. Cette spécificité de la démocratie suisse présente donc un intérêt particulier en regard du constat répété des limites de la démocratie représentative ${ }^{1}$. Le questionnement sur la participation démocratique a bénéficié, ces dernières années, de la relecture des réflexions de John Dewey (2002 [1927]) sur le processus de constitution d'un public politique en démocratie. S'appuyant sur sa théorie de l'enquête, J. Dewey propose non pas de définir ou de décrire le public politique, mais de préciser les conditions de sa constitution et les obstacles susceptibles de se présenter à elle. Il parait donc intéressant d'appliquer cette approche du public politique au cas de la démocratie semi-directe suisse.

Cette contribution analyse un scrutin de mars 2000 qui invitait les citoyens suisses à se prononcer sur une régulation très contraignante de la procréation assistée. Le volet empirique porte sur l'extrait d'un débat radiophonique pour décrire la façon dont l'émission constitue un public politique en préparation du vote. L'analyse de cet extrait suggère que le public en question est constitué comme n'étant pas tant concerné par la procréation assistée que par sa possible restriction par le biais de la régulation proposée. Il s'agit là d'une figure récurrente de la démocratie suisse, dans laquelle le citoyen est fréquemment appelé à se mobiliser pour refuser une régulation. Il s'agit, dans les termes de J. Dewey, de refuser de constituer le public d'un problème. La conclusion reprendra les résultats de cette analyse ainsi que d'autres cas d'initiatives populaires pour identifier un éventuel problème du public dans la démocratie semi-directe suisse. Il convient, préalablement à l'analyse, de présenter à grands traits l'apport de J. Dewey à la question de la démocratie.

1. Voir par exemple M. Callon et al., 2001.

Université de Fribourg (Suisse), alain.bovet@unifr.ch

Mots. Les langages du politique $\mathrm{n}^{\circ} 81$ juillet $2006 \bullet 35$ 


\section{Le public selon John Dewey}

Les considérations de J. Dewey sur le public politique sont indissociables de sa théorie de l'enquête ${ }^{2}$ (Dewey, 1993 [1938]). Il convient donc d'en indiquer les principaux aspects avant d'aborder la question du public. Pour J. Dewey, l'individualité se développe par le biais d'expériences. Celles-ci désignent des conduites qui résolvent pratiquement des situations vécues comme problématiques. C'est notamment la prise de conscience des conséquences de ses activités, et la réorientation de sa conduite qui en résulte, qui permet à l'individu de résoudre le caractère problématique d'une situation et de vivre ainsi une expérience $^{3}$.

La prise de conscience des conséquences d'activités est également au fondement de la constitution d'un public politique. J. Dewey part du principe que les activités humaines, les transactions, ont des conséquences, positives ou négatives, qui peuvent affecter - directement - ceux qui y participent ou - indirectement - ceux qui n'y participent pas. Les individus ou groupes indirectement affectés par des conséquences négatives ont intérêt à concevoir et à mettre en place des mesures destinées à prévenir ces conséquences. Selon les termes de J. Dewey (2002 [1927], p. 63), « le public consiste en l'ensemble de tous ceux qui sont tellement affectés par les conséquences indirectes de transactions qu'il est jugé nécessaire de veiller systématiquement à ces conséquences ». En d'autres termes, il est possible de parler de public dès lors que ce dernier vit une expérience dans le sens défini plus haut. Encore faut-il préciser en quoi consiste cette expérience.

J. Dewey ramène le processus de constitution du public à une enquête en précisant que celle-ci doit être libre et publique. La situation problématique doit en effet être soumise à une investigation qui, sur le modèle de l'enquête scientifique, doit se réorienter au fil de son développement. Il s'agit donc d'éviter de prédéfinir l'objet de l'enquête ou les modalités de son déroulement. Il s'agit, en particulier, de ne pas mettre prématurément un terme à l'enquête sur la base de principes normatifs qui lui préexistent. Cela n'implique cependant pas qu'il faille écarter toute forme de jugement. En effet, l'expérience d'une situation problématique ne peut pas ne pas procéder par des jugements, qu'il s'agisse d'identifier ce qui pose problème ou de choisir des solutions selon une hiérarchisation de finalités. Cependant, « les fins ou les valeurs prises en compte

2. Cette présentation rapide s’appuie sur les présentations de Joëlle Zask (1999, 2002) et sur le travail programmatique de Louis Quéré (2002a, 2002b) en vue d'une sociologie pragmatiste des problèmes publics.

3. Il faut préciser ici que la pensée pragmatiste de J. Dewey disqualifie aussi bien l'individualisme que le déterminisme. L'expérience permettant l'individualité est avant tout une transaction ou interaction entre un individu et un environnement (naturel ou social). Au terme de l'expérience, individu et environnement ont été transformés (J. Zask, 2002, p. 109). 
dans l'enquête sur un problème sont déterminées “dans et par le processus de l'enquête”, et non pas données d'avance » (L. Quéré, 2002a, p. 145).

La liberté de l'enquête porte donc de manière importante sur les normes et institutions de la vie sociale, dont la nécessité et la validité sont mises en suspens. Cette posture réflexive accorde de facto une importance centrale au discours, dans la mesure où la conduite de l'enquête consiste à thématiser et à expliciter des éléments de la situation qui peuvent ordinairement rester tacites. L'importance du discours découle également de la seconde contrainte, à savoir celle de la publicité de l'enquête et de ses résultats, qui apparait comme une condition nécessaire de la constitution du public. J. Dewey signale ainsi que «la communication des résultats de l'enquête sociale est la même chose que la formation de l'opinion publique » (J. Dewey, 2002 [1927], p. 176). II ne s'agit cependant en aucune façon de vulgariser un savoir expert :

La publicité des enquêtes, la "dissémination » des résultats dans des termes qui puissent être compatibles avec ceux du sens commun, n'ont pas pour fonction de faire acte d'une compétence devant laquelle chaque profane aurait le sentiment de devoir s'incliner, encore moins d'endoctriner, mais (1) de fournir au public les outils d'enquête sans lesquels il ne pourrait parvenir à identifier ses intérêts, et donc sans lesquels l'enquête ne pourrait être conclue, et (2) de soumettre à l'appréciation du public un discours à caractère hypothétique. (J. Zask, 1999, p. 280)

L'importance du discours et de la communication indique encore une fois que la constitution du public se rapporte à une expérience collective. Le public politique ne peut ainsi être réduit à une substance, à un principe ou à des institutions. Il doit être accompli ; il est en quelque sorte toujours à refaire. Cet aspect de la pensée de J. Dewey résonne avec l'approche procédurale de la publicité développée par Jürgen Habermas (1978), dans le sillage kantien de l'usage public de la raison. Le public de J. Dewey ne se limite cependant pas à la fonction de contrôle et de critique du pouvoir. La notion d'expérience implique chez lui un rôle plus actif, qui porte aussi bien sur l'identification du problème que sur la conception et la mise en place de solutions (J. Zask, 2002, p. 124).

Cette approche véritablement pragmatique permet de définir ce que J. Dewey considère comme le problème du public. Une société moderne complexe se compose d'innombrables collectivités qui, en tant que telles, ne constituent pas des publics. C'est seulement la prise de conscience d'un problème par ceux qui en sont indirectement affectés, et l'enquête engagée pour le résoudre, qui sont à même de faire émerger le public. Dans le cas de la démocratie suisse, il convient donc de distinguer la collectivité des citoyens suisses, que la Constitution dote d'un certain nombre de droits civiques, du public de tel problème particulier. En d'autres termes, il ne suffit pas de disposer d'institutions garantissant la participation politique de chacun pour faire émerger une démocratie ${ }^{4}$.

4. Cette remarque n'implique évidemment pas que de telles institutions ne soient pas nécessaires à 
Le travail démocratique de constitution d'un public ne peut donc jamais être achevé. Il peut seulement être amené à des clôtures provisoires. C'est ce statut très particulier qui conduit J. Dewey à parler de « problème du public ».

Les exigences de liberté et de publicité de l'enquête présentées plus haut dirigent donc l'attention vers le rôle crucial du discours, et en particulier du discours médiatique, dans le processus de constitution du public politique. Il semble dès lors judicieux de se pencher sur les discours médiatiques pour documenter et analyser ce processus. Il convient à ce stade de spécifier la nature des données et l'angle d'analyse.

\section{Le public du problème}

L'analyse porte sur un débat retransmis en direct par la première station radiophonique publique de Suisse romande le 23 février 2000 . Le débat prend place dans un processus spécifiquement suisse qu'il convient d'expliciter avant d'engager l'analyse. Le 12 mars 2000, les citoyens suisses étaient invités à se prononcer sur une initiative populaire dite «pour une procréation respectant la dignité humaine ».

L'initiative populaire est un droit civique qui permet aux citoyens de modifier la Constitution. Ceux qui souhaitent cette modification, les «initiants", récoltent 100000 signatures de citoyens approuvant leur projet. Le Parlement examine la légalité du projet et se prononce. L'initiative peut alors être soumise au vote des citoyens. Pour être acceptée, elle doit être approuvée par la majorité des votants, ainsi que celle des cantons. De telles votations ont lieu trois à quatre fois par an. Une seule votation comprend généralement plusieurs initiatives et référendums ${ }^{5}$. Par conséquent, les différents objets d'une votation sont susceptibles de faire l'objet de traitements publics et médiatiques fort divers.

L'initiative "pour une procréation respectant la dignité humaine » proscrivait toute technique de fécondation hors du corps de la femme, ainsi que celle du don de gamète, ce qui visait concrètement la fécondation in vitro et le don de sperme. Elle a donné lieu à une campagne de votation relativement restreinte, quoique non dénuée d'une certaine passion. Au terme de cette campagne, l'initiative a été rejetée par $73 \%$ des votants et tous les cantons suisses. Le caractère restreint de la campagne ainsi que le rejet sévère de l'initiative ont été rapportés, par les acteurs et commentateurs, à la position très minoritaire des « initiants », qui émanaient exclusivement des cercles chrétiens fondamentalistes.

la démocratie. Par ailleurs, la question de savoir quelle portion de la population a accès au droit de vote ne sera pas abordée ici. Rappelons simplement que les femmes suisses n'ont pu voter, sur le plan fédéral, qu'en 1971, et que les étrangers restent privés de droits civiques, sur le plan fédéral ainsi que dans de nombreux cantons et communes.

5. Le référendum est l'autre institution centrale de la démocratie directe. Il s'agit dans ce cas de récolter 50 ooo signatures contre une décision du Parlement qui est alors soumise au vote populaire. Il suffit de la majorité des votants pour annuler la loi visée par le référendum. 
Le titre de l'initiative indique bien la démarche des initiants. Il s'agit, d'une part, du constat que les techniques de procréation en cours ne respectent pas la dignité humaine et, d'autre part, de l'introduction d'une régulation de ce type d'activité par une modification de la Constitution. Il s'agit donc de pointer les conséquences des activités de procréation assistée, non seulement pour ceux qui y prennent part mais, grâce au principe général de dignité humaine, également pour ceux qui n'en sont qu'indirectement affectés. La démarche des initiants consiste donc, toujours selon les termes de J. Dewey, à constituer un public politique de la procréation assistée.

\section{Le public de l'initiative populaire}

Voyons maintenant comment le débat radiophonique appréhende concrètement cette problématique. L'analyse sera limitée à l'ouverture du débat. II s'agira de rendre compte de ce qui est accompli et reconnu comme signifiant par les participants, les journalistes ou les différents intervenants. Cette démarche s’inspire de l'analyse de conversation ethno-méthodologique (H. Sacks, 1992 ; M. Relieu et F. Brock, 1995). II ne sera cependant pas possible d'engager une analyse détaillée de la séquentialité des extraits retenus ${ }^{6}$. L'analyse procèdera essentiellement par des reformulations des propos échangés, l'objectif étant de rapporter ce qui est dit et fait, dans l'extrait retenu, à la problématique introduite plus haut. Commençons par l'introduction qui suit immédiatement le générique.

\section{Extrait 1 $^{7}$}

1 Générique

2 J1 Bonsoir. Le saviez-vous ? Depuis ce jour de 1978 où Louise Brown,

3 premier bébé éprouvette, vint au monde, six cent mille enfants sont nés

4 de la fécondation in vitro sur la planète. Et la petite Suisse n'est pas en

5 reste avec, chaque jour, deux bébés qui naissent de la procréation

6 médicalement assistée, la fameuse PMA. Une technique qui a fait ses

7 preuves ? À voir, puisque le 12 mars prochain, vous serez appelés à

8 vous prononcer sur une initiative populaire. Elle vise à interdire toute

9 procréation artificielle effectuée hors du corps de la femme. En clair,

6. Pour une analyse plus détaillée de ce débat radiophonique, voir A. Bovet (2001).

7. Les locuteurs sont indiqués par des lettres et des numéros qui renvoient respectivement aux fonctions dans le débat et à l'ordre d'entrée en scène. La lettre J désigne les journalistes, la lettre T les témoins et la lettre I les invités qui prennent part au débat radiophonique. Contrairement aux conventions en vigueur en analyse de conversation, la transcription est ici très proche de la langue écrite, à des fins de lisibilité. L'analyse fera cependant référence à des énoncés pour désigner les phrases de la transcription. 
10 elle vise à ce que soient interdits le don de sperme et la fécondation in

11 vitro. Initiative disproportionnée, disent les uns. Initiative qui

12 permettrait d'éviter les abus et les apprentis sorciers, disent les autres.

13 Forum rentre dans la mêlée et ouvre le débat en compagnie de Ghila

14 Zoutter, présidente d'Azote liquide, une association destinée à soutenir

15 les couples stériles, Pascale Della Santa, médecin favorable à

16 l'initiative, René Lysek, médecin-gynécologue, spécialiste en

17 procréation médicalement assistée, et, à Fribourg, le père dominicain

18 Roger Berthouzoz, professeur d'éthique et de théologie morale à

19 l'université de Fribourg.

20 Jingle

Dans cette riche introduction, le journaliste précise l'objet et l'enjeu du débat (1-13) avant de présenter les invités (13-19). Il présente l'initiative comme mettant en question la légitimité d'une technique d'assistance médicale à la procréation. Le journaliste souligne d'emblée, aux lignes 7-8, que la décision quant à la légitimité de cette technique sera prise par les récepteurs de l'émission (" vous »), qui sont implicitement identifiés aux citoyens suisses, par le biais de la notion d'« initiative populaire ». L'émission à venir apparait dès lors comme la préparation à une échéance démocratique. Aux lignes 11-12, le journaliste indique que le vote du 12 mars constituera le dénouement d'une confrontation bipolaire, mettant aux prises ceux qui jugent la proposition «disproportionnée » et ceux qui y voient une régulation salutaire de la médecine de procréation.

La séquence de présentation des quatre invités (13-19) recourt à de nombreuses catégorisations. Si la position des invités à l'égard de l'initiative n'est explicitée que pour Pascale Della Santa, celle des autres invités se déduit facilement des éléments de description fournis par le journaliste. Ce travail de catégorisation fait émerger une opposition qui vient recouvrir la bipolarité découlant de l'initiative. On trouve en effet dans le camp des opposants à l'initiative des invités qui sont présentés comme parties prenantes de la procréation assistée, avec une représentante des couples stériles et un médecin pratiquant et spécialisé. S'agissant du camp favorable à l'initiative, on a affaire à une femme médecin, sans autre précision que sa position vis-à-vis de l'initiative, ainsi qu'à un expert en matière d'éthique et de morale, dont le journaliste signale l'appartenance religieuse et académique.

Selon les termes de J. Dewey, le débat tel qu'il est configuré par le journaliste comprend, d'un côté, le camp de ceux qui prennent part aux activités de procréation assistée, les transactants, qui jugent l'initiative "disproportionnée » et, de l'autre côté, le camp de ceux qui prennent conscience des conséquences indirectes néfastes d'usages abusifs de la technique et prennent des mesures pour y remédier. La distinction entre les conséquences directes, éprouvées par les transactants, et les conséquences indirectes, qui n'affectent qu'indirecte- 
ment un public potentiel, prend ici tout son sens ${ }^{8}$. Elle permet au journaliste de confronter une position fondée sur une expérience à une position découlant de principes ou de croyances. En effet, les deux invités susceptibles de défendre l'initiative ne sont catégorisés par aucun élément rattachable à une expérience de la procréation assistée.

Les séquences suivantes du débat montrent que cette opposition spécifique continue de structurer pratiquement la conduite du débat. C'est notamment ce que montre l'extrait suivant, qui correspond à la séquence qui suit immédiatement celle qui vient d'être discutée.

\section{Extrait 2}

20 Jingle

21 J1 Alors en guise de préambule, pour entrer vraiment dans le débat, je

22 vous propose d'abord d'écouter ce témoignage recueilli par Nicolas

23 Vultier.

24 T1 On aurait aimé pouvoir faire comme tout le monde et avoir un enfant

25 normalement sans avoir à suivre toutes ces démarches et à avoir tous

26 ces frais. Mais si on nous avait empêchés de faire ça, aujourd'hui on se

27 porterait certainement très mal. Le fait d'avoir pu le faire et d'avoir pu

28 arriver à ce résultat-là pour nous est évidemment un grand bonheur.

Le journaliste lance le débat par le biais de cet enregistrement effectué par un autre journaliste et présenté comme un «témoignage ». Ce discours, prononcé par une voix masculine, se présente d'emblée comme le récit d'une expérience de la procréation assistée. Il établit, dans le premier énoncé (24-26), le caractère impératif du recours à cette technique. Cette formulation renvoie tacitement à la stérilité comme fatalité qui rompt la relation «normale " ( comme tout le monde », «normalement») entre le projet du couple et la procréation. Comme l'indiquent les deux énoncés suivants, la procréation assistée permet de restaurer cette relation «normale ». Le second énoncé (26-27) thématise, sous la forme d'une supposition, le programme d'action des initiants, pour en pointer les conséquences du point de vue de l'expérience du couple. L'obstacle à une restauration de la normalité apparait comme une cause de profonde souffrance. Le troisième énoncé (27-28) associe la restauration de la normalité, la procréation, à un sentiment qui lui est conventionnellement (« évidemment») associée, le « bonheur».

La forme du témoignage narratif permet donc de contraster deux cours d'action, notamment en fonction de leurs conséquences. Le premier consiste en la

8. Il ne s’agit pas ici de faire entrer les données dans les notions développées par J. Dewey, encore moins de faire du journaliste un Monsieur Jourdain de la philosophie pragmatiste. L'approche pragmatiste du public a une valeur descriptive qui est cependant indissociable de sa dimension normative. Ce point sera repris en conclusion. 
restauration d'une normalité qui entraine des conséquences favorables, voire impératives. Le second consiste en un obstacle hypothétique à cette restauration, dont les conséquences néfastes peuvent être anticipées avec certitude. Le témoignage institue donc une expérience directe de la procréation assistée qui fonde par ailleurs une condamnation de l'entreprise des initiants. La suite de la séquence de témoignage précise la relation des témoins à l'égard de l'initiative.

\section{Extrait 3}

29 J2 Et qu'une certaine frange de citoyens veuille interdire cela, ça vous

30 inspire quel sentiment?

31 T2 Beaucoup de tristesse, parce que c'est une atteinte à la liberté du

32 couple, de la famille et je trouve que ça regarde chaque personne, mais

33 personnellement. Et c'est une grande tristesse qu'on veuille nous

34 empêcher cette liberté de choix.

35 T1 Surtout qu'il faut bien comprendre qu'en l'occurrence les initiants

36 cherchent à nous empêcher de faire quelque chose qui fonctionne bien.

37 Quand on parle de manipulations génétiques ou d'OGM qui sont des

38 choses totalement différentes, on parle de choses qui vont venir et

39 qu'on connait mal. Quand on parle de PMA, on parle d'une pratique

40 qui a vingt ans derrière elle, qui fonctionne bien, qui ne coute pas

41 tellement à la société puisque c'est les couples eux-mêmes qui payent,

42 et qui nous rend heureux. Franchement, je vois pas bien les raisons là-

43 dedans d'empêcher ça. Maintenant, c'est clair qu'on s'est posé des

44 questions éthiques. Euh... mon épouse et moi sommes catholiques

45 pratiquants tous les deux. Il est clair que par rapport à notre foi on s'est

46 posé quelques questions. Est-ce que cette démarche-là était compatible

47 avec notre foi ou pas ? Et la réponse a manifestement été oui puisqu'on

48 a fait les démarches.

Aux lignes 29-30, la question ouverte du journaliste qui a recueilli le témoignage sollicite des témoins le "sentiment» suscité par le programme des initiants, désignés comme "une certaine frange de citoyens», donc rattachés à une position de l'espace politique. La réponse (31-34), énoncée par une voix féminine, articule le sentiment de «tristesse » à la violation d'un principe moral. En d'autres termes, le projet de régulation des initiants apparait comme un empiètement dommageable de la liberté de la personne, du couple et de la famille.

Le premier témoin reprend ensuite la parole et produit deux énonciations distinctes. Dans un premier temps, il développe un argumentaire en faveur de la préservation de la procréation assistée (35-43). Pour reprendre les termes de J. Dewey, le témoin présente l'enquête sur les risques de la procréation assistée comme ayant été menée à terme, contrairement à celle portant sur les OGM 
(37-40). Dans cette perspective, il ne peut être question, pour la procréation assistée, d'une éventuelle situation d'incertitude qui appellerait des investigations et des mesures de régulation. D'autre part, le témoin écarte l'argument du cout de la technique pour la collectivité, qui pourrait constituer une conséquence indirecte indésirable (40-41).

Dans un second temps, l'énonciation revient à une forme de témoignage (43-48). Le témoin rapporte qu'une réflexion a été menée au sein du couple sur la légitimité éthique, pour les catholiques, de la procréation assistée. Cette réflexion a débouché sur une évaluation positive du recours à la technique. Cette petite narration suggère entre autres choses que l'espace familial est l'espace légitime où une forme d'enquête doit être menée, et rejoint dans ce sens la réponse de la femme (31-34). Dans cette perspective, les mesures mises en œuvre pour résoudre la stérilité s'assimilent donc à des transactions au sein de l'association familiale. L'absence de conséquences indirectes rend simplement nulle et non avenue la conduite d'une enquête publique sur la question.

Ce témoignage à charge contre l'initiative ne consiste cependant pas seulement à présenter la procréation assistée comme une transaction relevant de la sphère privée. Il s'agit en effet également de faire de sa préservation un enjeu public. Le témoignage est dès lors à entendre comme une enquête sur les conséquences néfastes d'une éventuelle interdiction. Les témoins viennent raconter comment la situation problématique de la stérilité s'est transformée par le biais de la procréation assistée. La communication de cette expérience de couples stériles devenus parents vise ainsi à constituer un public susceptible de veiller à la préservation de sa possibilité.

C'est dans ce sens que le témoignage personnel du transactant de la procréation assistée vaut comme une prise de position politique, avec une visée de persuasion. Michel de Certeau a précisé comment le récit incarné se met au service du discours normatif susceptible de produire de la croyance :

Parce que la loi est déjà appliquée avec et sur des corps, « incarnée » en des pratiques physiques, elle peut s'en accréditer et faire croire qu'elle parle au nom du réel. [...] En d'autres termes, le discours normatif ne « marche » que si déjà il est devenu un récit, un texte articulé sur du réel et parlant en son nom, c'est-à-dire une loi historiée et historicisée, racontée par des corps. (M. de Certeau, 1990, p. 218)

On comprend, dès lors, qu'un témoignage soit légitime, non seulement «en préambule» mais "pour entrer vraiment dans le débat». C'est précisément dans cette logique que le journaliste qui anime l'émission accomplit la transition entre la séquence préenregistrée de témoignage et le débat en studio et en direct. L'intervention du journaliste transforme le témoignage antérieur en une question qui ouvre le débat. 


\section{Extrait 4}

49 J1 Pascale Della Santa, vous êtes opposée à la fécondation in vitro et c'est

50 votre droit. Mais un témoignage comme celui-là, est-ce qu'il n'ébranle

51 pas vos certitudes ? Euh... ces parents-là avaient un désir d'enfant très

52 fort, un désir qui a été exaucé, et c'est tant mieux, non?

53 l1 Mhm... Oui, je dois dire que, effectivement le désir d'enfant est tout à fait

54 légitime et je comprends tout à fait les couples qui désirent des enfants

55 et ne peuvent pas en avoir. Effectivement, je comprends que ça soit une

56 souffrance et certainement très grande. Cependant, je pense que le

57 moyen de la fécondation in vitro n'est pas, pour plusieurs raisons, un

58 bon moyen d'arriver à ces fins.

59 J1 Et on aura l'occasion de comprendre pourquoi, j'espère.

60 l1 Mhm... Tout à fait, oui.

La question du journaliste (49-52) est adressée à l'invitée présentée auparavant comme «médecin favorable à l'initiative ». La question la produit comme devant à la fois répondre au témoignage et répondre de l'initiative qu'elle défend ${ }^{9}$, et désigne une réponse préférentiellement positive. L'invitée commence par ratifier le caractère problématique de la situation des couples stériles (53-56). La formulation de son désaccord (56-58) consiste à mettre en question la fécondation in vitro comme mesure susceptible d'y remédier ${ }^{10}$. Une telle réponse ne met donc pas en question le cadrage mis en place dès l'introduction du débat. Il s'agit fondamentalement d'un problème de couples stériles.

La suite du débat radiophonique ne fait que reproduire et renforcer ce cadrage. Les transactants de la procréation assistée, la représentante de couples stériles et le médecin spécialisé témoignent de leur expérience, alors que les partisans de l'initiative sont requis de justifier leur opposition à la technique. Le fait que le débat soit ouvert par le biais du témoignage d'un couple stérile qui a eu un enfant grâce à cette technique ne relève donc ni de l'anecdote ni de la simple routine radiophonique. Une analyse de la presse suisse (Bovet, 2002) a montré que le témoignage des transactants a constitué la forme largement dominante de l'enquête suscitée par l'initiative « pour une procréation respectant la dignité humaine ». L'extrait de débat radiophonique analysé plus haut peut donc être considéré comme une réfraction du débat dans son ensemble ${ }^{11}$.

9. Dans la suite du débat, une question similaire devient une accusation morale fondée sur la catégorie de médecin: "Pascale Della Santa vous êtes aussi médecin, la stérilité est reconnue comme une maladie, en refusant finalement la procréation assistée est-ce que vous ne refusez pas de soigner des patients?»

10. L'invitée promet de fournir «plusieurs raisons » qui fondent sa position. La relance du journaliste à la ligne 59 suggère d'ailleurs que l'intelligibilité de cette position ne va pas de soi. On est donc loin de l'évidence propre au témoignage.

11. Cette opération métonymique n'est valide que pour des campagnes brèves, dont la configuration n'évolue pas ou peu. 
Il ressort de l'analyse et des éléments qui viennent d'être mentionnés que la procréation assistée a été publiquement définie et constituée comme une affaire privée, comme une transaction ne concernant que des couples et des médecins. Il convient cependant d'insister sur le fait que, si le déroulement contingent de la campagne n'a pas fait émerger le public visé par les initiants, il a néanmoins fait émerger un autre public. Les opposants à l'initiative sont en effet parvenus à faire de la préservation du statu quo un enjeu public. Il s'agit de faire d'une interdiction de la fécondation in vitro une situation problématique, qui appelle à ce titre l'opposition de l'ensemble de la collectivité suisse. Cette mobilisation s'est fondée sur le droit moral des couples stériles à disposer d'une thérapie médicale susceptible de réaliser leur projet parental. Selon les termes de J. Dewey, les opposants à l'initiative ont visé la prise de conscience des conséquences positives de la disponibilité de cette technique, et l'anticipation des conséquences négatives de son interdiction. Cette phase a été anticipée comme une condition préalable à l'action consistant à voter contre l'initiative. Le public ainsi constitué apparait dès lors davantage comme celui de l'initiative (sur la procréation assistée) que comme celui qui est favorable à cette pratique.

Précisons, pour conclure cette section, qu'il ne s'agit pas ici d'une interprétation rétrospective déduite du score médiocre de l'initiative, mais d'une observation des discours produits publiquement au cours de la campagne. C'est ce qui justifie l'analyse d'un débat radiophonique pour documenter la constitution contingente du public d'un problème.

Le vote de mars 2000 n'a rien d'un évènement de la démocratie suisse, ni même de l'histoire de la régulation des biotechnologies. Si la campagne a retenu une certaine attention médiatique, c'est essentiellement par le biais de l'indignation exprimée par des parents ayant recouru à la fécondation in vitro. Comme le montre la logique de l'ouverture du débat radiophonique analysé plus haut, la convocation du témoignage des parents a constitué l'essentiel de l'enquête menée autour de l'initiative " pour une procréation respectant la dignité humaine ». L'adoption majoritaire de cette perspective a eu pour effet de souligner le caractère intrusif de l'initiative dans la vie familiale.

L'initiative est donc loin d'avoir suscité la conduite d'une enquête, du moins selon la conceptualisation développée par J. Dewey. En effet, une telle enquête n'aurait pu être réduite au témoignage des parents ou des médecins. Elle aurait notamment initié un questionnement plus large sur les conditions anthropologiques de la filiation et de la production sociale de nouveaux membres de la société.

Le caractère restreint et inabouti de l'enquête effectivement conduite ne tient cependant pas au seul fait que c'est la perspective des opposants à 
l'initiative qui a été privilégiée. Les initiants proposaient en effet une perspective encore plus restreinte, qui consistait en la préservation du caractère sacré, selon eux, de l'acte sexuel conjugal. La médiation de la fécondation in vitro leur apparaissait comme la transgression de cette valeur. Il s'agit, dans cette perspective, d'une clôture de l'enquête encore plus arbitraire et prématurée que la prise en compte exclusive du témoignage des parents.

Au lendemain du vote, le caractère extrême de cette revendication a été largement invoqué pour expliquer le très net rejet de l'initiative, mais également l'échec de celle-ci à susciter un large débat sur la procréation assistée. Il s'agit là en effet de deux visées des initiatives populaires. Seule une initiative populaire sur dix est acceptée par la double majorité du peuple et des cantons. Il y a cependant des initiatives qui, bien que refusées, parviennent à faire prendre conscience d'une situation jugée problématique. Dans ce cas, le vote marque non pas la clôture de l'enquête, comme pour la procréation assistée en mars 2000, mais bien son initiation. L'enquête engagée est alors susceptible de déboucher sur des mesures plus ou moins proches de celles proposées par l'initiative.

C'est notamment le cas d'une initiative populaire déposée en 1993, qui proposait de fortes restrictions aux conditions d'application du génie génétique. Elle a suscité une importante campagne entre 1996 et juin 1998, lorsqu'elle a été rejetée par plus de deux tiers des votants et tous les cantons. Elle a cependant incontestablement infléchi la loi sur le génie génétique élaborée par le Parlement entre 2000 et $2003^{12}$. Cette initiative a joué un rôle essentiel dans la constitution d'un public du génie génétique en Suisse ${ }^{13}$.

On peut dès lors se demander ce qui fait l'intérêt de l'initiative « pour une procréation respectant la dignité humaine ». La réponse tient au caractère paradigmatique de cette initiative. Les campagnes de votation ont très fréquemment pour effet d'inviter le citoyen à se mobiliser pour renoncer à réguler une situation, voire même pour refuser d'entrer en débat sur cette régulation (C. Terzi, A. Bovet, 2005). C'est ainsi que des propositions de régulation plus stricte du secret bancaire, du commerce de l'armement ou de la recherche sur les cellules souches embryonnaires ont non seulement échoué au vote, mais également lors de la tentative de faire reconnaitre une situation problématique ${ }^{14}$. Indépendamment du fait que, comme indiqué plus haut, une large majorité des initiatives populaires connait cette fortune, il est crucial, pour une étude des espaces

12. C'est également le cas d'une initiative populaire déposée en 2000 qui proposait de très fortes restrictions du droit d'asile. Rejetée de justesse en 2002, cette initiative a vu presque tous ses articles adoptés depuis par le Parlement.

13. La différence entre le statut problématique des OGM et la légitimité de la procréation assistée est invoquée comme une évidence par le premier témoin dans l'extrait 3 (lignes 35-42).

14. Il faut mentionner ici les échecs répétés $(1974,1984,1999)$ de propositions de création d'une assurance-maternité - dont le principe est pourtant inscrit dans la Constitution - jusqu'à l'acceptation d'un projet de congé-maternité en 2004. 
publics démocratiques, de documenter aussi bien les échecs que les réussites des entreprises de publicisation de situations jugées problématiques. Il s'agit donc de spécifier comment la conduite pratique de l'enquête aboutit à faire un enjeu public de l'échec de l'initiative, plutôt que de son objet.

Il convient à ce point de revenir sur les perspectives ouvertes par les réflexions de J. Dewey sur l'enquête et sur le problème du public. Son approche ne peut être réduite à un vocabulaire descriptif. Sa conceptualisation de l'expérience interdit en effet de la limiter à de simples pratiques expérimentales. L'expérience est intrinsèquement orientée par la finalité d'identification et de résolution d'une situation problématique. Cette orientation de l'expérience, et a fortiori de l'enquête, introduit une dimension normative irréductible. En d'autres termes, la conception pragmatiste du public permet à la fois de rendre compte de la conduite de l'enquête et d'évaluer son degré d'aboutissement.

C'est dans cette perspective qu'a été menée l'analyse de l'ouverture du débat radiophonique présentée plus haut. Une analyse de l'organisation notamment interactive du discours permet de documenter la constitution de l'enjeu public accomplie par le débat radiophonique. Une analyse plus détaillée aboutirait à une formalisation de l'usage pratique de catégories telles que "parents », "enfants », "médecin », " souffrance», etc., ainsi qu'aux effets concrets de l'énonciation de telles catégories dans le déroulement séquentiel des échanges. Il s'agirait notamment de préciser comment des dispositifs de catégorisation qui, dans l'usage pratique, servent autant à décrire qu'à prescrire (Jayyusi, 1984), sont susceptibles d'ouvrir ou au contraire de clore l'investigation sur des situations jugées problématiques. Quel que soit le degré de détail de leurs analyses, les sciences sociales sont en mesure de contribuer à l'enquête ${ }^{15}$, notamment en cherchant à préciser les conditions susceptibles de favoriser ou d'entraver la conduite d'une enquête libre et publique.

\section{Références}

BOVETA., 2001, "L'expertise médicale comme discours public. Le débat sur la procréation médicalement assistée », Bulletin suisse de linguistique appliquée, $\mathrm{n}^{\circ} 74$, p. $129-148$.

2002, "La procréation comme enjeu public: Analyse des débats dans la presse suisse », V. Dasen éd., Regards croisés sur la naissance et la petite enfance, Fribourg, Éditions universitaires, p. 135-152.

15. J.Zask (2002, p. 127) précise que «seules les sciences sociales dont l'esprit est "interactionniste" peuvent aider à la reconstruction du public, et elles seules sont donc utiles, voire souhaitables, en démocratie ». "Interactionniste » implique ici la conception d'une détermination réciproque entre milieu et individualité. Sous cette acception, l'ethno-méthodologie peut être qualifiée d' « interactionniste ». 
et TERZIC., 2005, "La composante narrative des controverses politiques et médiatiques. Pour une analyse praxéologique des actions et des mobilisations collectives ", Réseaux, n 132, p. 111-132

CALLON M., LASCOUMES P. et BARTHE Y., 2001, Agir dans un monde incertain. Essai sur la démocratie technique, Paris, Le Seuil, $368 \mathrm{p}$.

CERTEAU M. (de), 1990, L'invention du quotidien, I. Arts de faire, Paris, Gallimard, 347 p.

DEWEY J., 1993 [1938], Logique. La théorie de l'enquête, Paris, PUF, 693 p.

2002 [1927], Le public et ses problèmes, Tours, Farrago/Éditions Léon Scheer, $207 \mathrm{p}$.

JAYYUSI L., 1984, Categorization and the Moral Order, Boston, Routledge \& Paul Kegan, $275 \mathrm{p}$.

HABERMASJ., 1978, L'espace public. Archéologie de la publicité comme dimension constitutive de la société bourgeoise, Paris, Payot, $324 \mathrm{p}$.

QUÉRÉ L., 2002a, «La structure de l'expérience publique d'un point de vue pragmatiste », D. Cefaï et I. Joseph éd., L'héritage du pragmatisme. Conflits d'urbanité et épreuves de civisme, La Tour d'Aigues, Éditions de l'Aube, p. 131-160.

_ 2002b, «Pour un calme examen des faits de société », B. Lahire éd., À quoi sert la sociologie ?, Paris, La Découverte, p. 79-94.

RELIEU M.et BROCKF., 1995, "L'infrastructure conversationnelle de la parole publique. L'analyse des réunions politiques et des interviews télédiffusées », Politix, $\mathrm{n}^{\circ} 31$, p. 77-112.

SACKS H., 1992, Lectures on conversation, Oxford, Basil Blackwell, $1520 \mathrm{p}$.

ZASK J., 1999, L'opinion publique et son double, II. John Dewey, philosophe du public, Paris, L'Harmattan, $314 \mathrm{p}$.

2002, "Éthiques et politiques de l'interaction. Le self-government à la lumière du pragmatisme », D. Cefaï et I. Joseph éd., op. cit., p. 107-129. 